EIMC: "INFECCIONES POR MICOBACTERIAS"

\title{
VACUNACIÓN FRENTE A TUBERCULOSIS
}

*Carlos Martín ${ }^{\mathrm{a}, \mathrm{b}, \mathrm{c},}$, Nacho Aguilo ${ }^{\mathrm{a}, \mathrm{b}}$, Jesus Gonzalo-Asensio ${ }^{\mathrm{a}, \mathrm{b}, \mathrm{c}}$

aGrupo de Genética de Micobacterias, Dpto. Microbiología, Medicina Preventiva y Salud Pública, Universidad de Zaragoza, C/ Domingo Miral s/n, 50009 Zaragoza, Spain.

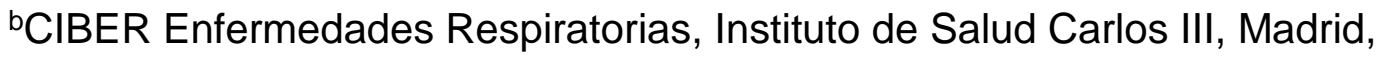
Spain.

'Servicio de Microbiología, Hospital Universitario Miguel Servet, ISS Aragón, 50009 Zaragoza, Spain.

* Correspondencia: Dpto. Microbiología, Medicina Preventiva y Salud Pública, Facultad de Medicina, Universidad de Zaragoza, C/ Domingo Miral s/n, 50009 Zaragoza, Spain. Phone: (+34) 9767617 59. E-mail: carlos@unizar.es. 


\title{
VACUNACIÓN FRENTE A TUBERCULOSIS
}

\begin{abstract}
:
BCG (Bacille Calmette-Guérin) vaccination is included in the immunization schedule for tuberculosis endemic countries with a global coverage at birth close to $90 \%$ worldwide. BCG was attenuated from Mycobacterium bovis almost a century ago, and provides a strong protection against disseminated forms of the disease, though very limited against pulmonary forms of tuberculosis, responsible for transmission.
\end{abstract}

Novel prophylactic tuberculosis vaccines are in clinical development either to replace BCG or to improve its protection against respiratory forms of the disease. There are limitations understanding the immunological responses involved and the precise type of long-lived immunity that new vaccines need to induce. MTBVAC is the first and only tuberculosis vaccine candidate based on live-attenuated Mycobacterium tuberculosis in clinical evaluation. MTBVAC clinical development plans to target tuberculosis prevention in newborns, as a BCG replacement strategy, and as secondary objective to be tested in adolescents and adults previous vaccinated with BCG.

Key words: BCG, tuberculosis vaccine, MTBVAC 


\section{Resumen:}

La vacunación con BCG (Bacilo Calmette-Guérin) está incluida en el calendario de inmunización al nacimiento en países endémicos de tuberculosis con una cobertura global cercana al 90\%. BCG tiene casi cien años de antigüedad, y está basada en una cepa atenuada de Mycobacterium bovis, proporcionando protección contra las formas diseminadas de la enfermedad, pero confiriendo una protección muy limitada contra las formas pulmonares de tuberculosis, responsables de su transmisión.

Diferentes vacunas profilácticas contra la tuberculosis se encuentran hoy desarrollo clínico para reemplazar a BCG, o para mejorar la protección en individuos ya vacunados con BCG. MTBVAC es la primera y única vacuna candidata basada en una cepa de Mycobacterium tuberculosis atenuada en evaluación clínica. Los planes de desarrollo clínico del MTBVAC se dirigen en primer lugar a la prevención de la tuberculosis en recién nacidos, para reemplazar a BCG y en segundo lugar en adolescentes y adultos.

Palabras clave: BCG, vacuna tuberculosis, MTBVAC 
La tuberculosis es la enfermedad infecciosa que más muertes causa en todo el mundo, superando al SIDA. Las últimas estimaciones de la Organización Mundial de la Salud (OMS) para el año 2016 fueron de 10,4 millones de nuevos casos de tuberculosis, causando cerca de 1,7 millones de muertes ${ }^{1}$. La tuberculosis está relacionada con la pobreza y agravada con la pandemia del VIH / SIDA. Actualmente, una de las mayores amenazas es la aparición de cepas resistentes a múltiples fármacos. 50 millones de personas están infectadas con cepas de $M$. tuberculosis multirresistentes, creando un reservorio para futuros casos de tuberculosis activa que dificultan enormemente el tratamiento ${ }^{1}$. Para conseguir un control efectivo de la tuberculosis necesitamos encontrar herramientas de diagnóstico más rápidas y fiables que las actuales, nuevos fármacos que permitan acortar la terapia, así como desarrollar nuevas vacunas más eficaces que la actual vacuna BCG contra las formas pulmonares de la enfermedad, responsables de su transmisión ${ }^{1}$.

La vacunación es una de las medidas más eficaces en términos de coste beneficio para luchar contra las enfermedades infecciosas. El Bacilo CalmetteGuérin (BCG) es actualmente la única vacuna autorizada y en uso contra la tuberculosis, con una cobertura cercana al 90\% en países con alta incidencia, a pesar de que su eficacia contra las formas respiratorias de la enfermedad es muy variable ${ }^{2,3}$ (Figura 1). 


\section{Historia de la BCG}

BCG es una vacuna atenuada derivada de Mycobacterium bovis, el agente etiológico de la tuberculosis en bóvidos ${ }^{4}$. BCG fue desarrollada por el médico Albert Calmette y el veterinario Camille Guérin entre los años 1908 y 1921 por subcultivo repetido de una cepa de $M$. bovis aislada de una vaca, siguiendo los principios de Pasteur para la construcción de vacunas atenuadas contra las enfermedades infecciosas. Tras 230 pases en el laboratorio durante 13 años, la cepa mostró su atenuación primero en terneros y posteriormente en cobayas y otros modelos animales ${ }^{5}$. BCG se introdujo por primera vez en clínica hace casi cien años, cuando en 1921 fue administrada oralmente a un bebé cuya madre había muerto de tuberculosis el día después de su nacimiento. El bebé no mostró efectos adversos a la vacunación con BCG y, lo que es más importante, no desarrolló tuberculosis. En aquellos años se eligió la vía oral de administración para BCG al considerarse el tracto gastrointestinal la vía natural de infección tuberculosa en lactantes y niños alimentados con leche no pasteurizada ${ }^{6}$. Entre 1921 y 1926 más de 50.000 niños fueron vacunados sin apenas efectos adversos. La mortalidad entre los niños vacunados fue del $1.8 \%$ comparada con una mortalidad superior al $25 \%$ en los niños no vacunados, mostrando su eficacia al disminuir la mortalidad infantil, no solo debida a tuberculosis ${ }^{5}$.

La cepa original BCG se distribuyó por todo el mundo, antes de su conservación por liofilización en los años 60. Dada la ausencia de métodos de conservación a largo plazo de microorganismos hasta esta fecha, cada laboratorio realizaba pases repetidos subcultivando la cepa original, dando 
lugar a la aparición de diferentes sub-cepas BCG y que fueron designadas por el nombre del laboratorio o del país donde eran subcultivadas, resultando en diferentes BCGs, con fenotipos heterogéneos. Actualmente seis cepas BCG son las más utilizadas en todo el mundo en los programas internacionales de inmunización: BCG Pasteur 1173 P2, BCG Danish 1331, BCG Glaxo 107, BCG Tokyo 172-1, BCG Rusia -I r y BCG Brasil 7.

La principal causa de la atenuación de BCG es la pérdida de la región diferencial 1 (RD1) asociada con la pérdida del factor de virulencia del antígeno secretado inmunodominante de $6 \mathrm{kDa}\left(\right.$ ESAT-6) ${ }^{4}$. El análisis genómico de las sub cepas de BCG muestra múltiples diferencias, incluyendo otras deleciones diferentes a RD1 que contribuyen a las variaciones fenotípicas entre ellas, con claras diferencias de atenuación, pero sin estar demostrado que contribuyan a diferencias en su eficacia ${ }^{8}$.

\section{Vacunación con BCG en la actualidad}

Desde 1974, la vacunación intradérmica con BCG al nacimiento ha sido incluida en el Programa Ampliado de Inmunización (EPI) de la OMS, resultando en más de 4.000 millones de vacunas administradas en todo el mundo y aproximadamente 200 millones de vacunas son administrada cada año. La concentración de bacterias vivas en las vacunas oscila entre 50.000 y 3 millones por dosis dependiendo de la cepa BCG utilizada ${ }^{9}$.

En Europa algunos países como Irlanda se recomiendan actualmente la vacunación con BCG, mientras que en otros países como Francia o Portugal se 
recomendaba la vacunación con BCG en el pasado, pero no en la actualidad (Figura 2). La información sobre las políticas y prácticas de vacunación con BCG en todo el mundo se puede consultar en la página: http://www.bcgatlas.org/ y las específicas para Europa en http://vaccineschedule.ecdc.europa.eu/Pages/Scheduler.aspx.

Hoy la OMS recomienda la vacunación con BCG en todos los recién nacidos en países con alta incidencia de TB. BCG no está recomendada para bebés con infección conocida por $\mathrm{VIH}$ u otra inmunodeficiencia. En países con alta prevalencia de TB y VIH, es importante tener precaución cuando se administra BCG de forma rutinaria debido al riesgo de enfermedad por BCG diseminada en bebés infectados con VIH. BCG está recomendada en los lactantes asintomáticos nacidos de madres con estado VIH desconocido. En la actualidad, la recomendación es la inmunización rutinaria de BCG durante la infancia, hasta que se pueda implementar un programa de despistaje sistemático de VIH.

En países con baja incidencia de TB, se puede considerar la inmunización con BCG en niños $\leq 5$ años procedentes de países endémicos. También si el niño está continuamente expuesto a un paciente no tratado o que no responda al tratamiento y que tiene tuberculosis pulmonar infecciosa y no es posible la separación del paciente. También cuando el niño está continuamente expuesto a un paciente que tiene tuberculosis pulmonar infecciosa causada por cepas de M. tuberculosis resistentes a isoniazida y rifampina, y la separación del paciente infeccioso no es posible ${ }^{10}$. 
La Unión Internacional contra la Tuberculosis y Enfermedades Respiratorias (IUATLD) y la OMS han establecido una serie de criterios según los cuales recomiendan que un país cambie de una política de vacunación sistemática con BCG a la vacunación selectiva de grupos de alto riesgo. La recomendación de suspender la vacunación sistemática con BCG en un país determinado sería en caso de que si existe un sistema de notificación de casos de tuberculosis eficaz y la tasa de notificación anual de tuberculosis pulmonar con baciloscopia inferior al 5 por 100.000 o si el riesgo anual de infección tuberculosa es < $0.1 \%$. También si la tasa anual de notificación de meningitis tuberculosa es <1 por cada 10 millones de habitantes en los últimos 5 años ${ }^{10}$.

La recomendación de vacunación con BCG para adultos que viajan a áreas endémicas con alta exposición a tuberculosis multirresistente continúa siendo controvertida. Dado el riesgo potencial de fracaso del tratamiento antituberculoso, y a la baja tasa de complicaciones relacionadas con la vacunación con BCG en individuos inmunocompetentes, se podría recomendar la administración de BCG en individuos no vacunados, tuberculina o IGRA negativos expuestos a tuberculosis multirresistente. Son necesarios más estudios para contrastar la eficacia protectora de BCG en el contexto de la exposición a la tuberculosis multirresistente en adultos ${ }^{10}$.

La seguridad de la vacuna BCG ha sido probada ampliamente después de más de 4 mil millones de unidades administradas en todo el mundo desde 1921. BCG es una vacuna muy reactogénica, pero muy segura y bien tolerada, 
produciendo una reacción local en el lugar de la inyección ${ }^{9}$. Después de 2-6 semanas de recibir la vacuna, aparece una pequeña pápula que aumenta de tamaño y que en la mayoría de los casos progresa a una úlcera. Los nódulos linfáticos cervicales y axilares puede aumentar de tamaño temporalmente. Después de un período de aproximadamente 3 meses, aparecerá una cicatriz, que será permanente.

\section{Contraindicaciones de BCG}

BCG al nacimiento estaría contraindicada en niños desnutridos y recién nacidos prematuros con un peso al nacer de menos de $2.5 \mathrm{~kg}$. Dado que la vacuna BCG es una vacuna viva también está contraindicada en mujeres embarazadas y pacientes oncológicos o inmunocomprometidos que sufran una inmunodeficiencia congénita o adquirida por fármacos inmunosupresores, radiación o por infección con VIH. Tampoco se recomienda administrar la vacuna BCG si el paciente ha sido tratado con antibióticos durante los últimos 30 días.

BCG también está contraindicada en personas con tuberculosis activa o con prueba de tuberculina positiva o con infección tuberculosa, aunque estudios recientes han demostrado que BCG es segura en personas con infección tuberculosa latente ${ }^{11}$.

\section{Administración con otras vacunas o productos}

BCG mejora las respuestas de células T y B a otros antígenos vacunales y puede ser co-administrada con cualquier otra vacuna (incluidas otras vacunas 
vivas) sin haber sido descritos problemas mayores. La BCG mejora las respuestas de las citocinas Th1 y Th2 a antígenos no relacionados y aumenta la respuesta de anticuerpos en la vacunación oral contra la polio.

La única limitación sería la necesidad de administración en diferentes sitios anatómicos y no se recomienda administrar ninguna otra vacuna en la misma extremidad dentro de los 3 meses posteriores a la administración de BCG ${ }^{10}$.

\section{Respuesta inmune a BCG}

Sabemos que una inmunidad celular es importante para el control de la tuberculosis, pero no existe un marcador inmunológico que se pueda correlacionar con protección frente a la enfermedad. La respuesta inmune a la inmunización primaria con BCG ha sido evaluada en diferentes estudios en niños que demuestran que hay una inducción asociada a BCG de células T polifuncionales CD4 + y CD8 + interferón (IFN $\gamma$ ) - +, interleucina (IL) -2+, factor de necrosis tumoral $(\mathrm{TNF} \alpha)+$, pero no ha sido posible demostrar una correlación de protección ${ }^{12}$.

\section{Eficacia variable de BCG}

A pesar de su gran cobertura, el grado de protección de BCG contra las formas respiratorias de tuberculosis sigue siendo objeto de debate ${ }^{3}$. En lactantes, está demostrado que BCG ofrece protección contra las formas diseminadas de tuberculosis (miliar y meníngea) ${ }^{13}$, mientras que en adolescentes y adultos vacunados al nacer, la eficacia de BCG contra las formas pulmonares de la enfermedad es muy variable, dependiendo de la edad de vacunación y del 
estudio ${ }^{2}$, considerándose que la inmunidad de BCG disminuye con el tiempo y se recomienda su administración lo más cercana posible al nacimiento ${ }^{14}$.

Estudios recientes muestran que BCG, a pesar de conferir una protección moderada, dura más de lo que previamente seconsideraba, hasta al menos 20 años ${ }^{15}$. Hay muchas y variadas hipótesis sobre por qué hay tal variación en la eficacia de BCG; sin embargo, para ninguna se ha encontrado una respuesta definitiva. Se cree que la sobre-atenuación de BCG por la pérdida de antígenos inmunodominantes durante el proceso de subcultivos repetidos podría ser una de las razones de estas diferencias, como dijimos anteriormente, pero no ha sido demostrado ${ }^{8}$. Numerosos ensayos clínicos sugieren que la revacunación con BCG no mejora su eficacia, lo que podría deberse a una inmunidad preexistente por la infección por micobacterias ambientales no tuberculosas, previas a la vacunación que podrían conducir a un enmascaramiento (masking) o bloqueo (blocking) de la re-vacunación con BCG ${ }^{16}$.

\section{Beneficios no específicos de la vacunación con BCG}

Un número creciente de estudios muestran que las vacunas vivas atenuadas reducen la morbilidad y mortalidad infantil, lo cual es un hecho admitido hoy en día por la OMS ${ }^{17}{ }^{18}$. En algunos países de África Occidental BCG reduce la mortalidad neonatal mortalidad en más del $40 \%$, principalmente mediante la prevención de sepsis e infecciones respiratorias y fiebre ${ }^{19}$. Estudios en países donde la mortalidad infantil es muy baja, como en España, se han descrito los efectos beneficiosos inespecíficos de la vacunación con BCG al nacimiento, concluyendo que disminuye la hospitalización por infecciones respiratorias y 
sepsis, no relacionadas con tuberculosis a través de un efecto no específico ${ }^{20}$ 21. Recientemente se ha demostrado que BCG puede inducir resistencia inespecífica a patógenos a través de una reprogramación epigenética de los monocitos $^{22} 23$. Estos efectos se han descrito para células del sistema inmune innato como macrófagos y células NK (natural killer) ${ }^{24} 25$. Las vías metabólicas presentan un papel esencial en la inmunidad en monocitos humanos, regulada por mecanismos epigenéticos a nivel de la organización de la cromatina, poniendo de manifiesto el potencial terapéutico que la modulación de estas vías puede tener durante la vacunación ${ }^{26}$. Cualquier nueva vacuna con mejor eficacia contra las formas respiratorias de tuberculosis deberá conservar estos efectos inespecíficos de BCG.

\section{Investigación y Desarrollo de nuevas vacunas contra la tuberculosis}

Dada la falta de protección de BCG contra las formas respiratorias de tuberculosis, en los últimos 20 años se ha realizado un enorme esfuerzo en la investigación y el desarrollo de nuevas vacunas contra la tuberculosis ${ }^{27}$. Tras el descubrimiento de miles de candidatos y de que cientos hayan pasado a ensayos preclínicos en modelos animales, solo unas decenas pasan a ser ensayados en estudios clínicos en humanos. Hay diferentes etapas que cada candidato a vacuna necesita realizar en ensayos clínicos Fase I, Fase II, de Fase III, para alcanzar la autorización de comercialización. En Europa, la investigación impulsada por los diferentes Programas Marco de la Comisión Europea, ha hecho posible cientos de candidatos han pasado a ensayos preclínicos y varios, están actualmente en ensayos clínicos en humanos ${ }^{28}$. 
Las vacunas más eficaces hoy en uso contra diferentes enfermedades infecciosas inducen anticuerpos neutralizantes confiriendo una inmunidad protectora, mientras que para otras enfermedades como el SIDA, la malaria o la tuberculosis es necesaria una fuerte respuesta de la inmunidad celular ${ }^{29}$.

Una de las mayores dificultades para encontrar vacunas eficaces contra estas enfermedades es que no existe un marcador inmunológico que pueda predecir la eficacia de una nueva vacuna en estudio, lo que obliga a que sean probadas en largos y costosos estudios de eficacia con miles de voluntarios (Fase IIb y Fase III) en países endémicos con alta incidencia de estas enfermedades tras obtener datos robustos de seguridad e inmunogenicidad en ensayos previos con decenas (Fase I) y luego con centenas (Fase II) de voluntarios sanos ${ }^{27}$.

\section{Primer ensayo clínico de eficacia de una nueva vacuna contra la}

\section{tuberculosis}

Tras más de 10 años de ensayos clínicos previos la vacuna MVA85A, desarrollada por la Universidad de Oxford y liderada por la Dra. Helen McShane realizó el primer ensayo de eficacia de una vacuna contra la tuberculosis en un país endémico, en Worcester, Sudáfrica ${ }^{30}$. La vacuna MVA85A fue concebida para aumentar la inmunidad en niños previamente vacunados con BCG a los que se les administró el virus de la vacuna modificado de Ankara (MVA) al que se había introducido el gen que codifica para el antígeno mayor de tuberculosis Ag85A. El estudio de eficacia Fase Ilb consistió en un estudio de doble-ciego, placebo-control en niños sanos con edades comprendidas entre los 4 y 6 meses, no infectados por VIH y que 
habían recibido previamente BCG al nacimiento, con un seguimiento cada 3 meses durante más de tres años. Un total de 2.797 niños fueron vacunados (1.399 con MVA85A y 1.398 con un placebo). El resultado mostró que 32 niños (2\%) de los 1.399 vacunados con BCG+ MVA85A 39 fueron diagnosticados de tuberculosis y 39 niños (3\%) de los 1.398 vacunados con BCG + placebo. La diferencia entre los dos grupos no fue significativa y la interpretación del resultado del estudio fue la ausencia de eficacia de MVA85 ${ }^{30}$. Este resultado fue considerado por las agencias financiadoras del estudio como un fracaso.

Para la comunidad científica que trabajamos en vacunas contra la tuberculosis, por el contrario, fue un gran paso adelante en la investigación de nuevas vacunas, ya que este ensayo abre el camino a nuevos estudios de eficacia. El ensayo de eficacia de MVA85 fue el primero en casi 100 años después de BCG en los años 20 del siglo XX. El estudio en Worcester fue coordinado por la Iniciativa Sudáfrica para vacunas contra la tuberculosis (SATVI). Años después de este estudio la comunidad científica sigue aprendiendo y sacando conclusiones sobre la inmunología de la enfermedad. Tras 3 años de seguimiento de los niños del estudio, se estudió el QuantiFERON (QFT) y el riesgo de enfermedad en colaboración de los equipos de SATVI y de la Universidad de Oxford. Estos resultados ${ }^{30}$. muestran que tanto los niños QFT(menor de $0.35 \mathrm{UI} / \mathrm{ml}$ ) o QFT+ pero con menos de $4 \mathrm{UI} / \mathrm{ml}$ el riesgo de desarrollar tuberculosis es bajo mientras que los niños QFT+ con más de 4 $\mathrm{Ul} / \mathrm{ml}$ el riesgo es alto, resultados que pueden acelerar estudios con otras vacunas ${ }^{31}$ 


\section{Diversidad de los nuevos candidatos a Vacuna en ensayos clínicos}

Tras la publicación de los resultados del primer estudio de eficacia en niños, las Agencias de financiación y los investigadores se plantearon como diversificar los candidatos de vacunas contra la tuberculosis ya que la mayoría de los candidatos actuales son vacunas con poca diversidad de antígenos (Ag85A o Ag85B, ESAT6..) diseñadas para potenciar una inmunidad previa mediada por células $\mathrm{T}{ }^{27}$. En los últimos 10 años, dos organizaciones se encargan de coordinar la búsqueda de nuevos candidatos a vacunas en distintas fases preclínicas y en acelerar la aplicación en humanos de las nuevas vacunas obtenidas en el laboratorio. Por un lado, TBVI, la iniciativa europea (TBVI acrónimo en inglés de Iniciativa Europea para la vacuna contra la Tuberculosis) y por otro lado la estadounidense AERAS (apoyada por la Fundación Bill y Melinda Gates).

\section{¿Cuál sería la población que debemos vacunar?}

Tras los resultados negativos del primer estudio de eficacia, también se planteó cuál era la población prioritaria para vacunar: ¿niños? o ¿adultos?. Los estudios de modelización muestran que el mayor impacto de una nueva vacuna contra la tuberculosis sería claramente en adolescentes y adultos, donde la transmisión de la enfermedad es mayor ${ }^{32}$, ya que aunque la incidencia de la enfermedad es muy importante en niños menores de 5 años, no son comunes las formas respiratorias, responsables de la transmisión ${ }^{33}$.

En nuestra opinión, los recién nacidos sanos representan a la población más sensible y sin inmunidad preexistente a BCG o micobacterias ambientales, para 
ensayar la eficacia de una nueva vacuna dado que el grupo de mayor edad puede conducir a posibles efectos de enmascaramiento y bloqueo a la vacunación ${ }^{16}{ }^{34}$. Por ello pensamos que en primer lugar se debería estudiar la eficacia en recién nacidos y una vez comprobada la eficacia de una nueva vacuna, vacunar a adolescentes y adultos donde el impacto sobre la tuberculosis será mayor dado que son las formas pulmonares las responsables de esta transmisión ${ }^{34}$ (Figura 3).

\section{Nuevas vacunas contra la tuberculosis en ensayos clínicos}

Actualmente hay 12 vacunas contra la tuberculosis en ensayos clínicos ${ }^{1}$ (Figura 4) . La mayoría de estas vacunas se basan en subunidades en los que los antígenos de $M$. tuberculosis se expresan como proteínas recombinantes que se formulan con diferentes adyuvantes o bien expresadas por virus recombinantes que se utilizan como vehículos para la administración de antígenos.

Algunas de estas vacunas hoy en clínica están basadas en micobacterias inactivadas y han sido diseñadas como "vacunas terapéuticas" buscando disminuir los tiempos de tratamiento en las personas infectadas con tuberculosis latente, o bien reducir la probabilidad de recurrencia después de la finalización del tratamiento ${ }^{35}$. Las dos vacunas terapéuticas en desarrollo clínico comprenden o bien formas inactivadas de micobacterias no tuberculosas como son VaccaeTM en estudios Fase III de eficacia, o bien fragmentos de pared de $M$. tuberculosis vehiculizados en liposomas como es el caso de la vacuna RUTI® actualmente en Fase lla de ensayos clínicos ${ }^{36}$. 
Las estrategias de vacunación clásicas buscan prevenir la enfermedad y es lo que denominamos vacunas profilácticas. En tuberculosis se han desarrollado dos estrategias para el desarrollo de vacunas preventivas, la primera buscaría potenciar la acción de la actual vacuna BCG reforzando su inmunidad y la segunda reemplazar a la actual vacuna BCG por una vacuna más eficaz.

Dado que la mayor parte de la población de países endémicos de tuberculosis ha sido vacunada previamente con BCG al nacer y lo que se busca con las vacunas de refuerzo con antígenos de $M$. tuberculosis específicos potenciando a BCG. Una de estas vacunas en ensayos clínicos, DAR-901 se basa en una bacteria no tuberculosa inactivada por calor ${ }^{37} 1$ mientras las otras vacunas subunidades incluyen uno o unos pocos antígenos proteicos específicos de $M$. tuberculosis que administrados bien vectores virales o bien formulados con adyuvantes ${ }^{1}$ (Figura 5).

Son tres las vacunas subunidades que usan vectores virales utilizando distintos virus y diferentes rutas de administración. Una se encuentra en la Fase Ila, es la vacuna TB / FLU-04L, que utiliza el virus de la gripe atenuado como vector recombinante expresando los antígenos Ag85A y ESAT6 y es administrada por vía mucosal. Otras dos se encuentran en Fase I de ensayos clínicos, son las vacunas Ad5 Ag85 y ChadAdOx18A-MVA85A. La vacuna Ad5 Ag85A está siendo desarrollada por la Universidad de McMaster en Canada. Consiste en un vector de adenovirus de serotipo 5 que expresa Ag85A que se administra por vía intramuscular. La vacuna ChAdOx185A, desarrollada por la Universidad 
de Oxford, se basa en un adenovirus de simio y MVA85A (virus de viruela modificado) que expresan Ag85A por vía sistémica y mucosal.

Otras cuatro vacunas subunidades utilizan distintos adyuvantes para la administración de antígenos de tuberculosis. M72/AS01E desarrollada por Glaxo se encuentra en Fase IIb de ensayos clínicos, y otras tres en Fase IIA, como son las vacunas H4:IC31 y H56:IC31 desarrolladas por el Statem Serum Institut (SSI) de Copenhague y la vacuna ID93 + GLA-SE, desarrollada por Infectious Disease Research Institute (IDRI) en los Estados Unidos.

M72 I AS01E es una vacuna de subunidad que combina dos antígenos de $M$. tuberculosis (32A y 39A) con el adyuvante (AS01E). Está siendo probado en un ensayo de eficacia de Fase IIb en adultos VIH negativos infectados con $M$. tuberculosis en Kenia, Sudáfrica y Zambia. H4: IC31 es una vacuna de refuerzo BCG que contiene una proteína de fusión de Ag85B y TB10.4, formulada con adyuvante IC31.

La vacuna IC31. H56: IC31 combina tres antígenos de M. tuberculosis (Ag85B, ESAT-6 y Rv2660c) y el adyuvante IC31. La vacuna ID93 + GLA-SE contiene cuatro antígenos de M. tuberculosis asociados con virulencia (Rv2608, Rv3619 y Rv3620) o latencia (Rv1813) y el adyuvante GLA-SE.

En las vacunas profilácticas que buscan reemplazar a BCG, se considera que la inmunidad que confieren las vacunas vivas induce respuestas inmunes de memoria específicas de larga duración, que no se obtienen con las vacunas de 
subunidades. Este efecto podría estar relacionado con la persistencia o replicación restringida in vivo observada para otras vacunas vivas humanas (por ejemplo, polio, sarampión, fiebre amarilla) ${ }^{38}$.

Dos son las vacunas que buscan reemplazar a BCG en el nacimiento que se basan en vacunas vivas atenuadas derivadas de $M$. bovis BCG, la vacuna VPM1002 desarrollada por el Instituto Max Planck de Berlín en Fase II a de ensayos clínicos o de M. tuberculosis, MTBVAC en Fase I de ensayos clínicos.

La vacuna VPM1002 se basa en BCG recombinante (rBCG) expresando la listeriolisina de Listeria monocytogenes y con una deleción del gen que codifica para la ureasa C están diseñados para mejorar la eficacia de BCG mediante la inserción de otros genes. Actualmente se está llevando a cabo un ensayo de Fase II en Sudáfrica para evaluar la seguridad e inmunogenicidad de la vacuna en recién nacidos expuestos o no expuestos al $\mathrm{VIH}^{39}$.

\section{Estudios clínicos de eficacia de MTBVAC: una vacuna viva atenuada del patógeno humano}

Actualmente no sabemos cuáles son las respuestas inmunológicas ni el tipo preciso de inmunidad duradera que una nueva vacuna contra la tuberculosis debería inducir. Por ello, para comprender la inmunología de la protección contra la enfermedad necesitamos conseguir vacunas efectivas. Dado que, como hemos dicho anteriormente, la mayoría de las vacunas se basan principalmente en un pequeño número de antígenos con diferentes sistemas de administración, con la idea de aumentar la diversidad de las vacunas 
candidatas, elegimos construir una vacuna viva atenuada a partir de un patógeno de origen humano, buscando imitar la infección natural ya que cerca del $80 \%$ de los infectados por tuberculosis latente no sufre una reinfección por M. tuberculosis ${ }^{40}$ y que al estar la cepa atenuada se elimina el riesgo de desarrollar tuberculosis. Además, el hecho de partir de una bacteria viva hace que se mantenga todo el repertorio antigénico completo del patógeno humano.

La vacuna MTBVAC es la única vacuna viva atenuada derivada de un aislamiento de $M$. tuberculosis en ensayos clínicos cumpliendo los requisitos exigidos en el consenso de Ginebra 4546 (Figura 6). Desarrollada por la Universidad de Zaragoza y el Instituto Pasteur con el apoyo de la Iniciativa de Vacuna contra la TB (TBVI). MTBVAC muestra una respuesta de células T más duradera a diferentes de antígenos de $M$. tuberculosis no presentes en BCG ${ }^{42}$, y confiere una mejor protección que BCG en diversos modelos animales 4344 . El desarrollo industrial y posterior desarrollo clínico lo realiza la Biofarmaceutica española Biofabri con una amplia experiencia en vacunas vivas atenuadas (CZ veterinaria), evitando el subcultivo que podría dar origen a diferentes cepas como ocurrió con BCG en el pasado.

MTBVAC se construyó a partir de un aislado humano de $M$. tuberculosis, que pertenece al linaje 4 (Euroafricano-americano), uno de los linajes más extendidos de M. tuberculosis. La vacuna consiste en dos mutaciones estables mediante la deleción de los genes de virulencia phoP y fadD26. Por tanto, MTBVAC contiene todos los genes presentes en las cepas de M. tuberculosis, incluidos los genes ausentes en $M$. bovis y delecionados durante los 
subcultivos de BCG. MTBVAC ha completado el primer ensayo clínico en humanos ${ }^{41}$. Los resultados de la Fase la en Adultos mostraron un perfil de seguridad robusta para MTBVAC en las tres dosis probadas hasta llegar a una dosis de $10^{5}$ equivalente a la utilizada actualmente con BCG ${ }^{47}$. Los estudios de inmunología mediante estimulación de la sangre de los participantes con BCG o MTBVAC mostraron una correlación dosis/respuesta de linfocitos CD4 polifuncionales. En los voluntarios en los que se administró la misma dosis de MTBVAC que de BCG, el número de individuos que respondieron tras 4 semanas de la vacunación, fue mayor en el grupo vacunado con MTBVAC.

La prueba de Elispot para ESAT6 y CFP10, que se utiliza para diferenciar individuos vacunados con BCG e infectados por tuberculosis, fue negativo para todos los vacunados con MTBVAC ${ }^{47}$ a los 7 meses después de la vacunación, pero se pudo observar un tendencia de la respuesta específica contra CFP10ESAT 6 en los participantes vacunados con MTBVAC en comparación con BCG ${ }^{42}$.

Una de las principales diferencias entre las vacunas BCG y MTBVAC es que un gran número de epítopos ausentes en BCG está presente en MTBVAC. Podemos decir que MTBVAC posee un 50\% más de epítopos reconocidos por células T humanas en comparación con BCG. Además, MTBVAC secreta más proteínas del complejo Ag85 que BCG $^{48}$ y a diferencia de BCG, MTBVAC secreta Ag85B. MTBVAC produce los principales antígenos delecionados en todas las cepas de BCG y presentes en la región RD1, como son ESAT6 y CFP10 42. Nuestros estudios recientes muestran la importancia del 
reconocimiento de estos antígenos por parte del huésped, y sólo los ratones cuyos MHC son capaces de reconocer ESAT6 / CFP10 están mejor protegidos frente a la infección, lo que demuestra que la eficacia protectora de MTBVAC se asocia con la respuesta mediada por células T a CFP10 / ESAT6 que pueden ser importantes para la inmunidad ${ }^{49}$.

El plan de desarrollo clínico para MTBVAC considera como primer objetivo la vacunación en recién nacidos dado que esta población no ha estado previamente expuesta a micobacterias ambientales ni vacunados previamente con BCG. Es importante remarcar la importancia de la vacunación de esta población dada la alta incidencia de tuberculosis en bebés menores de 5 años (muy similar a los adolescentes) ${ }^{33}$.

En la actualidad, la Fase lb en bebés se está finalizando en SATVI en Worcester, donde los recién nacidos fueron vacunados con dosis crecientes de MTBVAC y la vacunación con la dosis más alta finalizó en septiembre de 2016. Tras más de un año de seguimiento no se han descrito efectos indeseables mayores relacionados con la vacuna y los resultados de inmunología se esperan que estén listos a principios de 2018 (ClinicalTrials.gov Identifier: NCT02729571).

\section{Futuros estudios clínicos de eficacia de MTBVAC}

La unión europea a través de su programa European and Developing Countries Clinical Trials Partnership (EDCTP) aprobó recientemente la financiación de la Fase Ila en bebes. Esta Fase Ila, de búsqueda de dosis óptima en recién 
nacidos (VIH no expuesto, BCG sin tratamiento previo, sin exposición familiar conocida a TB). Con el objetivo principal: evaluar la seguridad y reactogenicidad del MTBVAC en dosis crecientes, en comparación con la vacuna BCG, y evaluar la inmunogenicidad de MTBVAC con las tres dosis crecientes en comparación con la vacuna BCG. El objetivo secundario es: evaluar la dinámica de la conversión y reversión QFT inducida por la vacuna MTBVAC. Un total de 99 recién nacidos serán vacunados $(75+24)$. Cohorte 1 : 25 con cada dosis diferente de MTBVAC y 24 con BCG. En 2 sitios adicionales: Instituto Pasteur Madagascar y Saint Louis Senegal, se estudiará la epidemiología para preparar futuros ensayos de eficacia.

El segundo objetivo para el desarrollo clínico de MTBVAC son los adolescentes I adultos. El estudio aprobado recientemente por el Congreso de los Estados Unidos y el National Institutes of Health (NIH), coordinado por AERAS se iniciará en 2018. El ensayo consistirá en un estudio de seguridad, inmunogenicidad y escalada de dosis en adultos con y sin infección latente de tuberculosis en también en Sudáfrica (ClinicalTrials.gov Identifier: NCT02933281). El objetivo principal es estudiar la seguridad y reactogenicidad de MTBVAC comparado con BCG en adultos y como objetivo secundario: estudiar la inmunogenicidad del MTBVAC a cuatro dosis crecientes medidos por análisis de sangre completa y tasas de conversión de QuantiFERON en adultos QFT-negativos.

Las vacunas vivas, como MTBVAC, son sólidos candidatos para "reemplazar a BCG" y para usarse donde más se necesitan, es decir, en los países con 
mayor incidencia de TB, una vez que podamos demostrar que MTBVAC es mejor que BCG. El desafío que tenemos por delante es la planificación de ensayos clínicos de eficacia en países con alta incidencia de tuberculosis, que podría acelerarse en gran medida mediante la identificación de marcadores de protección.

Agradecimientos: Este trabajo ha sido financiado por el Ministerio de Economía y Competitividad (BIO2014-5258P) y la Unión Europea dentro del programa H2020 (TBVAC2020 643381) y European \& Developing Countries Clinical Trials Partnership (EDCTP) RIA2016V-1637.

Conflicto de Interés: CM, JG and NA son inventores de patentes relacionadas con vacuna contra la tuberculosis cuyo titular es la Universidad de Zaragoza y de las cuales la empresa Biotecnológica española de Biofabri es licenciataria en exclusividad. 


\section{Referencias}

1. WHO. WHO GLOBAL TUBERCULOSIS REPORT 2017. WHO, editor. 19 de octubre de $2017 ;: 1-262$.

2.

Mangtani P, Abubakar I, Ariti C, Beynon R, Pimpin L, Fine PEM, et al. Protection by BCG vaccine against tuberculosis: a systematic review of randomized controlled trials. CLIN INFECT DIS. febrero de 2014;58(4):470-80.

3.

Zwerling A, Behr MA, Verma A, Brewer TF, Menzies D, Pai M. The BCG World Atlas: A Database of Global BCG Vaccination Policies and Practices. PLoS Med. Public Library of Science; 22 de marzo de 2011;8(3):e1001012.

4.

Brosch R, Gordon SV, Garnier T, Eiglmeier K, Frigui W, Valenti P, et al. Genome plasticity of BCG and impact on vaccine efficacy. Proc Natl Acad Sci USA. 27 de marzo de 2007;104(13):5596-601.

5.

Gheorghiu M, Lagranderie M, Balazuc A-M. Vaccines: A Biography. Artenstein AW, editor. New York, NY: Springer New York; 2009. 16 p.

6.

Kaufmann SHE, Weiner J, Reyn von CF. Novel approaches to tuberculosis vaccine development. Int J Infect Dis. marzo de 2017;56:263-7.

7.

Ho MM, Southern J, Kang H-N, Knezevic I. WHO Informal Consultation on standardization and evaluation of BCG vaccines Geneva, Switzerland 22â€"23 September 2009. Elsevier Ltd; 8 de octubre de 2010;28(43):6945-50.

8.

Zhang L, Ru H-W, Chen F-Z, Jin C-Y, Sun R-F, Fan X-Y, et al. Variable Virulence and Efficacy of BCG Vaccine Strains in Mice and Correlation With Genome Polymorphisms. Mol Ther. febrero de 2016;24(2):398-405.

9.

Lambach DP. WHO INFORMATIVE SHEET BCG. 2012 jun pp. 1-5.

10.

Martinón-Torres F, Martin C. Tuberculosis Vaccines. En: Pediatric Vaccines and Vaccinations. Cham: Springer International Publishing; 2017. pp. 149-60.

11.

Hatherill M, Geldenhuys H, Pienaar B, Suliman S, Chheng P, Debanne SM, et al. Safety and reactogenicity of BCG revaccination with isoniazid pretreatment in TST positive adults. Elsevier Ltd; 30 de junio de 2014;32(31):3982-8.

Kagina BMN, Abel B, Scriba TJ, Hughes EJ, Keyser A, Soares A, et al. Specific T Cell Frequency and Cytokine Expression Profile Do Not Correlate with Protection against Tuberculosis after Bacillus Calmette-Guérin Vaccination of Newborns. American Journal of Respiratory and Critical Care Medicine. 15 de octubre de 2010;182(8):1073-9.

13. Trunz BB, Fine $P$, Dye C. Effect of BCG vaccination on childhood tuberculous meningitis and miliary tuberculosis worldwide: a meta-analysis and assessment of cost-effectiveness. The Lancet. 14 de abril de 2006;367(9517):1173-80.

14. Mcshane $\mathrm{H}$. Understanding BCG is the key to improving it. CLIN INFECT DIS. febrero de 2014;58(4):481-2.

15. Mangtani P, Nguipdop-Djomo P, Keogh RH, Sterne JA, Abubakar I, Smith PG, et al. The duration of protection of school-aged BCG vaccination in England: a population -based case-control study. Int J Epidemiol. 2017.

16.

Barreto ML, Pilger D, Pereira SM, Genser B, Cruz AA, Cunha SS, et al. Causes of variation in BCG vaccine efficacy: Examining evidence from the BCG REVAC cluster randomized trial to explore the masking and the blocking hypotheses. 
Elsevier Ltd; 20 de mayo de 2014;:1-6.

17. Aaby $\mathrm{P}$, Benn CS. Beneficial non-specific effects of oral polio vaccine (OPV): Implications for the cessation of OPV? CLIN INFECT DIS. 12 de abril de 2017.

18. Benn CS, Fisker AB, Whittle HC, Aaby P. Revaccination with Live Attenuated Vaccines Confer Additional Beneficial Nonspecific Effects on Overall Survival: A Review. EBioMedicine. Elsevier; agosto de 2016;10:312-7.

19.

Benn CS, Netea MG, Selin LK, Aaby P. A small jab - a big effect: nonspecific immunomodulation by vaccines. Trends in Immunology. Elsevier Ltd; 1 de septiembre de 2013;34(9):431-9.

20.

de Castro MJ, Pardo-Seco J, Martinón-Torres F. Nonspecific (Heterologous) Protection of Neonatal BCG Vaccination Against Hospitalization Due to Respiratory Infection and Sepsis. CLIN INFECT DIS. 1 de junio de 2015;60(11):1611-9.

21. Iglesias MJ, Martin C. Editorial Commentary: Nonspecific Beneficial Effects of BCG Vaccination in High-income Countries, Should We Extend Recommendation of BCG Vaccination? CLIN INFECT DIS. 13 de mayo de 2015;60(11):1620-1.

Kleinnijenhuis JJ, Quintin JJ, Preijers FF, Joosten LABL, Ifrim DCD, Saeed SS, et al. Bacille Calmette-Guerin induces NOD2-dependent nonspecific protection from reinfection via epigenetic reprogramming of monocytes. Proc Natl Acad Sci USA [Internet]. National Acad Sciences; 22 de octubre de 2012;109(43):17537-42. Recuperado a partir de: http://eutils.ncbi.nlm.nih.gov/entrez/eutils/elink.fcgi?dbfrom=pubmed\&id=2298808 $2 \&$ retmode $=$ ref\&cmd=prlinks

Aaby PP, Benn CSC. Saving lives by training innate immunity with bacille Calmette-Guerin vaccine. Proc Natl Acad Sci USA. 22 de octubre de 2012;109(43):17317-8.

24. Netea MG, Quintin J, van der Meer JWM. Trained Immunity: A Memory for Innate Host Defense. Cell Host Microbe. Elsevier Inc; 19 de mayo de 2011;9(5):355-61.

25.

Netea MG, Joosten LAB, Latz E, Mills KHG, Natoli G, Stunnenberg HG, et al. Trained immunity: A program of innate immune memory in health and disease. Science. 22 de abril de 2016;352(6284):aaf1098-8.

26.

Arts RJW, Carvalho A, La Rocca C, Palma C, Rodrigues F, Silvestre R, et al. Immunometabolic Pathways in BCG-Induced Trained Immunity. Cell Reports. 2016;17(10):2562-71.

Marinova D, Gonzalo-Asensio J, Aguilo N, Martin C. Recent developments in tuberculosis vaccines. Expert review of vaccines. diciembre de 2013;12(12):143148.

28.

Kaufmann SHE, Dockrell HM, Drager N, Ho MM, Mcshane H, Neyrolles O, et al. TBVAC2020: Advancing Tuberculosis Vaccines from Discovery to Clinical Development. Front Immunol. 2017;8:1203.

29.

Rappuoli R, Aderem A. A 2020 vision for vaccines against HIV, tuberculosis and malaria. Nature. Nature Publishing Group; 17 de mayo de 2011;473(7348):463-9.

Tameris MD, Hatherill M, Landry BS, Scriba TJ, Snowden MA, Lockhart S, et al. Safety and efficacy of MVA85A, a new tuberculosis vaccine, in infants previously vaccinated with BCG: a randomised, placebo-controlled phase $2 \mathrm{~b}$ trial. The Lancet. febrero de 2013. 

Serial QuantiFERON testing and tuberculosis disease risk among young children: an observational cohort study. The Lancet Respiratory Medicine. 2017;5(4):28290. transmission of Mycobacterium tuberculosis in high burden settings. The Lancet Infectious Diseases. 2016;16(2):227-38.

Blaser N, Zahnd C, Hermans S, Salazar-Vizcaya L, Estill J, Morrow C, et al. Tuberculosis in Cape Town: An age-structured transmission model. Epidemics. marzo de 2016;14:54-61.

Arregui S, Sanz J, Marinova D, Martin C, Moreno Y. On the impact of masking and blocking hypotheses for measuring the efficacy of new tuberculosis vaccines. PeerJ. 2016;4:e1513.

Cardona P-J. The Progress of Therapeutic Vaccination with Regard to Tuberculosis. Front Microbiol. 2016;7:1536.

Nell AS, D'lom E, Bouic P, Sabaté M, Bosser R, Picas J, et al. Safety, Tolerability, and Immunogenicity of the Novel Antituberculous Vaccine RUTI: Randomized, Placebo-Controlled Phase II Clinical Trial in Patients with Latent Tuberculosis Infection. Borrow R, editor. PLOS ONE. 26 de febrero de 2014;9(2):e89612. development. Tuberculosis. Elsevier Ltd; 1 de agosto de 2016;99(Supplement 1):S16-S20. Vaccination Against Tuberculosis With Whole-Cell Mycobacterial Vaccines. J INFECT DIS. 1 de septiembre de 2016;214(5):659-64.

Nieuwenhuizen NE, Kulkarni PS, Shaligram U, Cotton MF, Rentsch CA, Eisele B, et al. The Recombinant Bacille Calmette-Guérin Vaccine VPM1002: Ready for Clinical Efficacy Testing. Front Immunol. 2017;8:1147.

Andrews JR, Noubary F, Walensky RP, Cerda R, Losina E, Horsburgh CR. Risk of progression to active tuberculosis following reinfection with Mycobacterium tuberculosis. CLIN INFECT DIS. marzo de 2012;54(6):784-91.

Arbués A, Aguilo Jl, Gonzalo-Asensio J, Marinova D, Uranga S, Puentes E, et al. Construction, characterization and preclinical evaluation of MTBVAC, the first liveattenuated $M$. tuberculosis-based vaccine to enter clinical trials. Vaccine. 1 de octubre de 2013;31(42):4867-73.

42. Aguilo N, Gonzalo-Asensio J, Alvarez-Arguedas S, Marinova D, Gomez AB, Uranga $S$, et al. Reactogenicity to major tuberculosis antigens absent in BCG is linked to improved protection against Mycobacterium tuberculosis. Nature Communications. Nature Publishing Group; 14 de julio de 2017;8:16085.

43.

Marinova D, Gonzalo-Asensio J, Aguilo N, Martin C. MTBVAC from discovery to clinical trials in tuberculosis-endemic countries. Expert review of vaccines. junio de 2017;16(6):565-76.

Aguilo N, Uranga S, Marinova D, Monzon M, Badiola J, Martin C. MTBVAC vaccine is safe, immunogenic and confers protective efficacy against Mycobacterium tuberculosis in newborn mice. Tuberculosis (Edinburgh, Scotland). enero de 2016;96:71-4. 

live mycobacterial vaccines: the Geneva consensus on essential steps towards clinical development. 2005. pp. 3753-61.

46. Walker KB, Brennan MJ, Ho MM, Eskola J, Thiry G, Sadoff J, et al. The second Geneva Consensus: Recommendations for novel live TB vaccines. 2010. pp. 2259-70.

47. Spertini F, Audran R, Chakour R, Karoui O, Steiner-Monard V, Thierry A-C, et al. Safety of human immunisation with a live-attenuated Mycobacterium tuberculosis vaccine: a randomised, double-blind, controlled phase I trial. The Lancet Respiratory Medicine. diciembre de 2015;3(12):953-62.

48. Solans L, Gonzalo-Asensio J, Sala C, Benjak A, Uplekar S, Rougemont J, et al. The PhoP-dependent ncRNA Mcr7 modulates the TAT secretion system in Mycobacterium tuberculosis. PLoS Pathog. mayo de 2014;10(5):e1004183.

49. Carpenter C, Sidney J, Kolla R, Nayak K, Tomiyama H, Tomiyama C, et al. A side-by-side comparison of $T$ cell reactivity to fifty-nine Mycobacterium tuberculosis antigens in diverse populations from five continents. Tuberculosis (Edinburgh, Scotland). diciembre de 2015;95(6):713-21.

50. Murray RA, Mansoor N, Harbacheuski R, Soler J, Davids V, Soares A, et al. Bacillus Calmette Guerin Vaccination of Human Newborns Induces a Specific, Functional CD8 T Cell Response. The Journal of Immunology. 2006;177(8):564751. 
Cobertura de la vacuna BCG en el año 2016

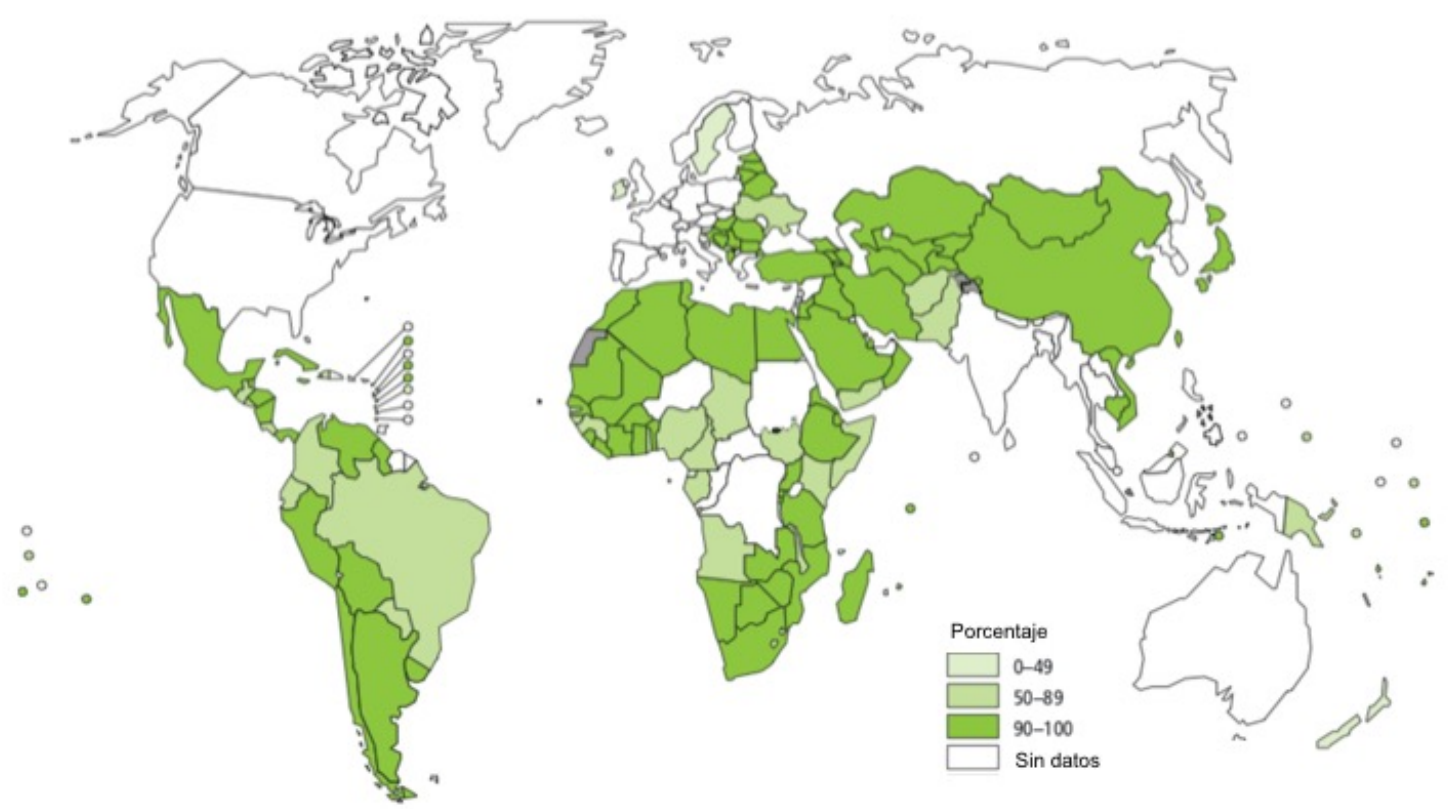

Figura 1 Cobertura de la vacuna BCG en el año 2016. Mapa indicando el porcentaje de vacunación con BCG en los diferentes países. Fuente WHO Global Tuberculosis Report 2017. 
Recomendación de vacunación con BCG

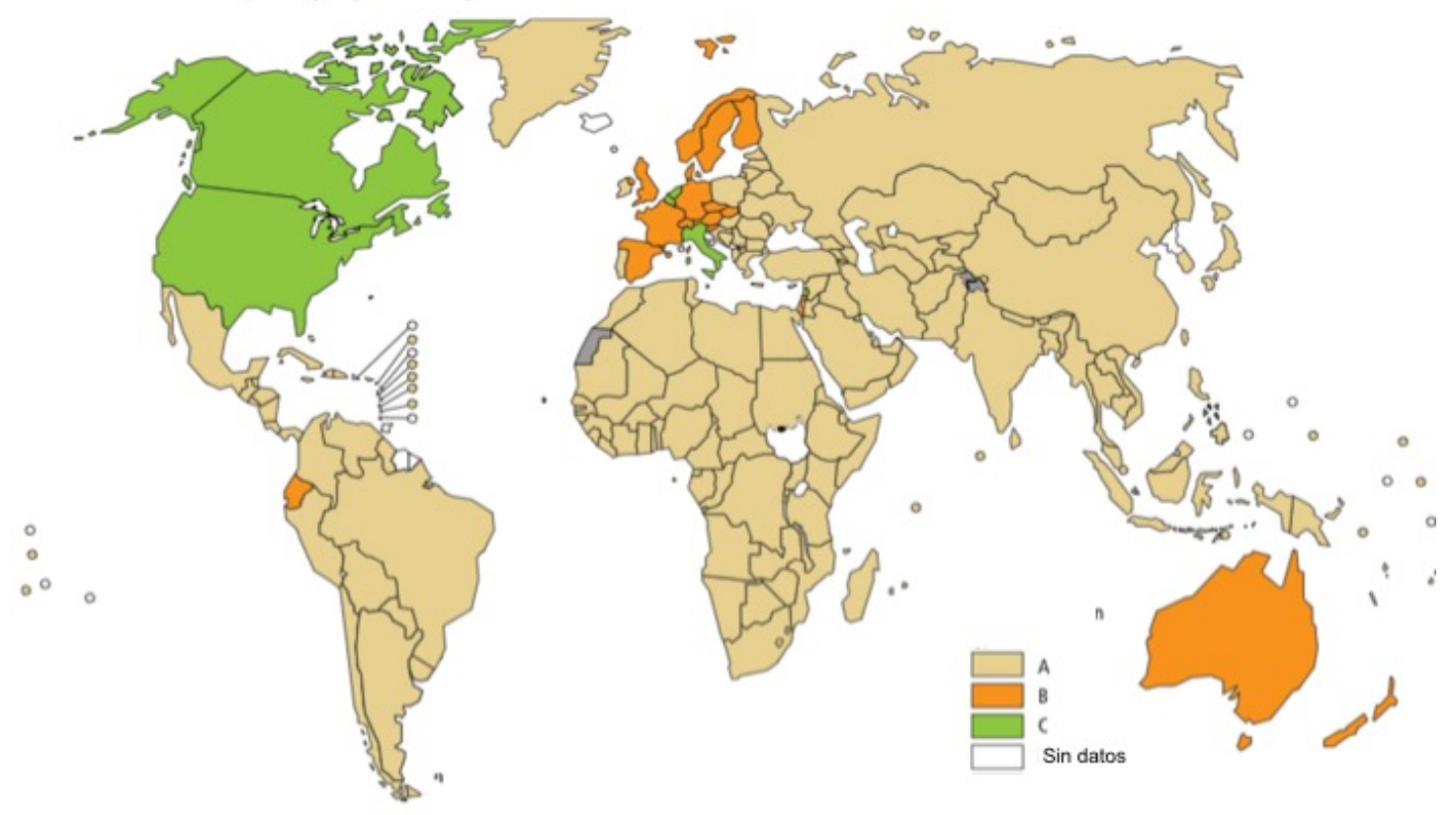

A.- BCG incluida en el programa de vacunación

B.- Vacunación con BCG en el pasado

C.- BCG indicada para grupos especificos

Figura 2 Recomendación de vacunación con BCG. Mapa representando en beige los países que incluyen BCG en su programa de vacunación (A), en naranja los países que vacunaron con BCG, pero no lo hacen actualmente (B) y en verde los países que BCG solo está indicada en grupos específicos de población (C). Fuente WHO Global Tuberculosis Report 2017. 


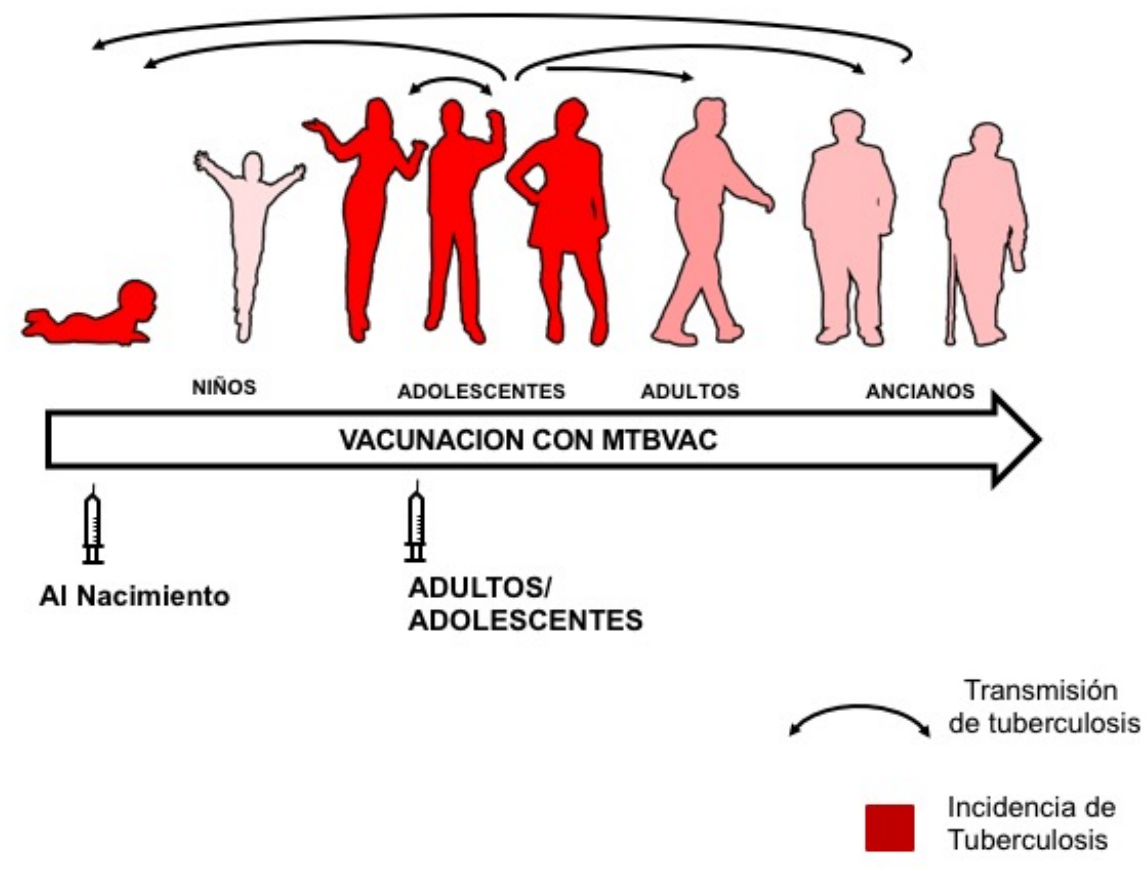

Figura 3 Transmisión de tuberculosis por grupos de edades y diferentes estrategias de vacunación. El papel de los adolescentes y adultos en la transmisión de la enfermedad está indicado por flechas. En color rojo los grupos con mayor incidencia de tuberculosis, niños menores de 5 años y adolescentes. La introducción de una nueva vacuna al nacimiento permitiría la protección de los niños desde el nacimiento haciendo posible estudiar su eficacia en una población naive no expuesta anteriormente a micobacterias ni previamente con BCG. La vacunación en población adolescente y adulta tendría un mayor impacto en la transmisión de la enfermedad. 


\section{VACUNAS CONTRA LA TUBERCULOSIS EN ENSAYOS CLINICOS}

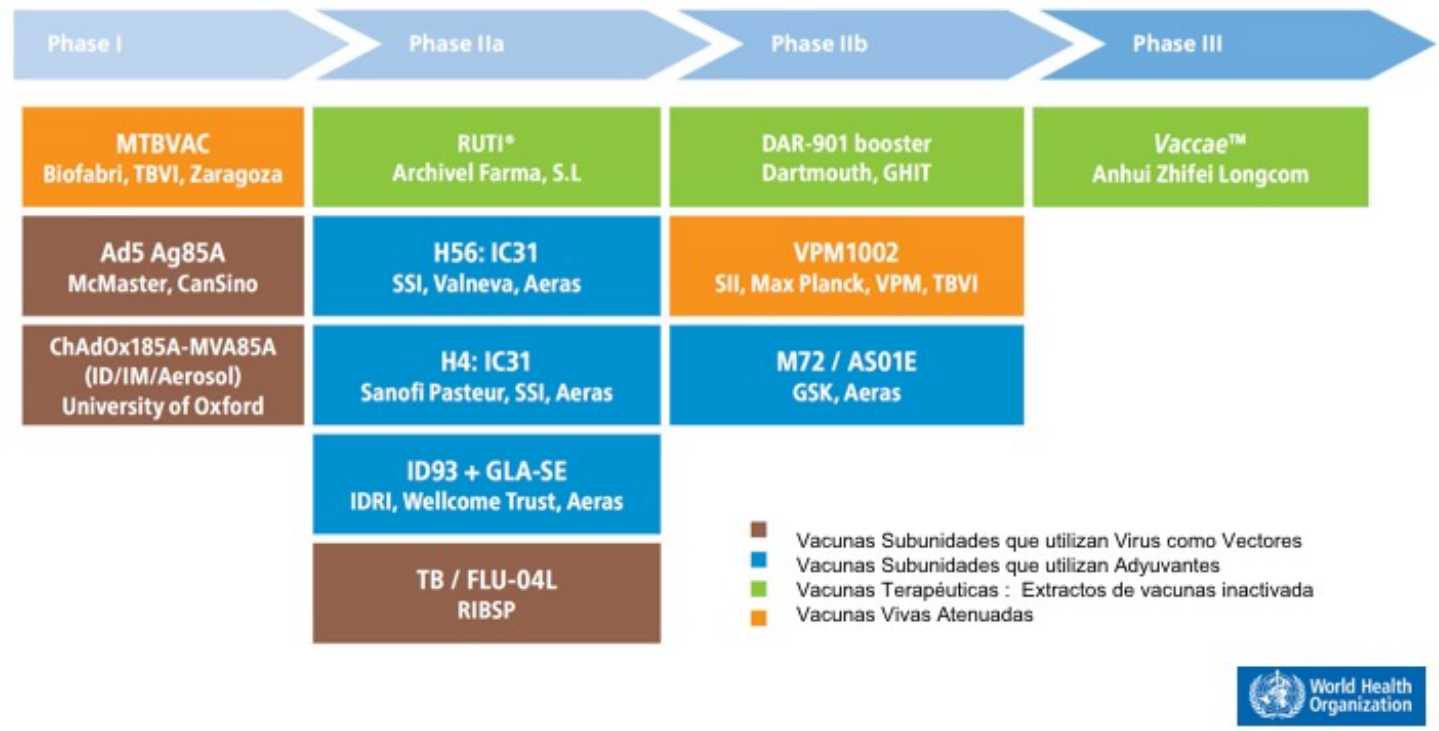

igura 4 Vacunas contra la tuberculosis en ensayos clínicos. En marrón, las vacunas subunidades que utilizan virus como vectores, en azul, Las vacunas subunidades que utilizan adyuvantes para su administración. En verde vacunas terapéuticas compuestas de extractos de vacunas inactivada y en naranja las vacunas vivas atenuadas. Fuente WHO Global Tuberculosis Report 2017. 


\section{VACUNAS SUBUNIDADES}

\section{VIRUS como VECTORES de antígenos de tuberculosis}

\begin{tabular}{|llc|}
\hline MVA85A & Virus de la Vacuna expresando Ag85A & $\begin{array}{c}\text { Primer estudio de eficacia } \\
\text { (Tameris et al 2013) }\end{array}$ \\
Ad5Ag85A & Adenovirus serotipo 5 expresando Ag85A & Fase I \\
ChAdox185A/MVA85A & Adenovirus de simio + MVA85A & Fase I \\
TB/Flu -04L & Virus de la gripe & Fase lla \\
\hline
\end{tabular}

Proteínas de fusión + ADYUVANTES

\begin{tabular}{llll}
\hline H4 & Ag85B/TB10.4 & IC31 & Fase lla \\
H56 & Ag85B/TB10.4/Rv2660c & IC31 & Fase lla \\
ID93 +GLA-SE & Rv1813/Rv2608/Rv3619/Rv3620 & GLA-SE & Fase lia \\
M72/ AS01E & Rv1196/Rv0125 & SO2 & Fase llb \\
\hline
\end{tabular}

Figura 5 Vacunas subunidades en ensayos clínicos. Las vacunas subunidades buscan en individuos previamente vacunados con BCG reforzar con antígenos de M. tuberculosis aumentar su protección. Pueden utilizar diferentes virus como vectores, como Poxvirus (MVA) o adenovirus de distintos orígenes ( $\mathrm{Ad}$ o $\mathrm{ChA}$ ) o el virus de la gripe. Otras vacunas subunidades utilizan distintos adyuvantes (IC31, GLA-SE o SO2) para potenciar el efecto inmunogeno de las proteínas de $M$. tuberculosis. Para cada candidato subunidad se indica la fase de desarrollo clínico en que se encuentra. 


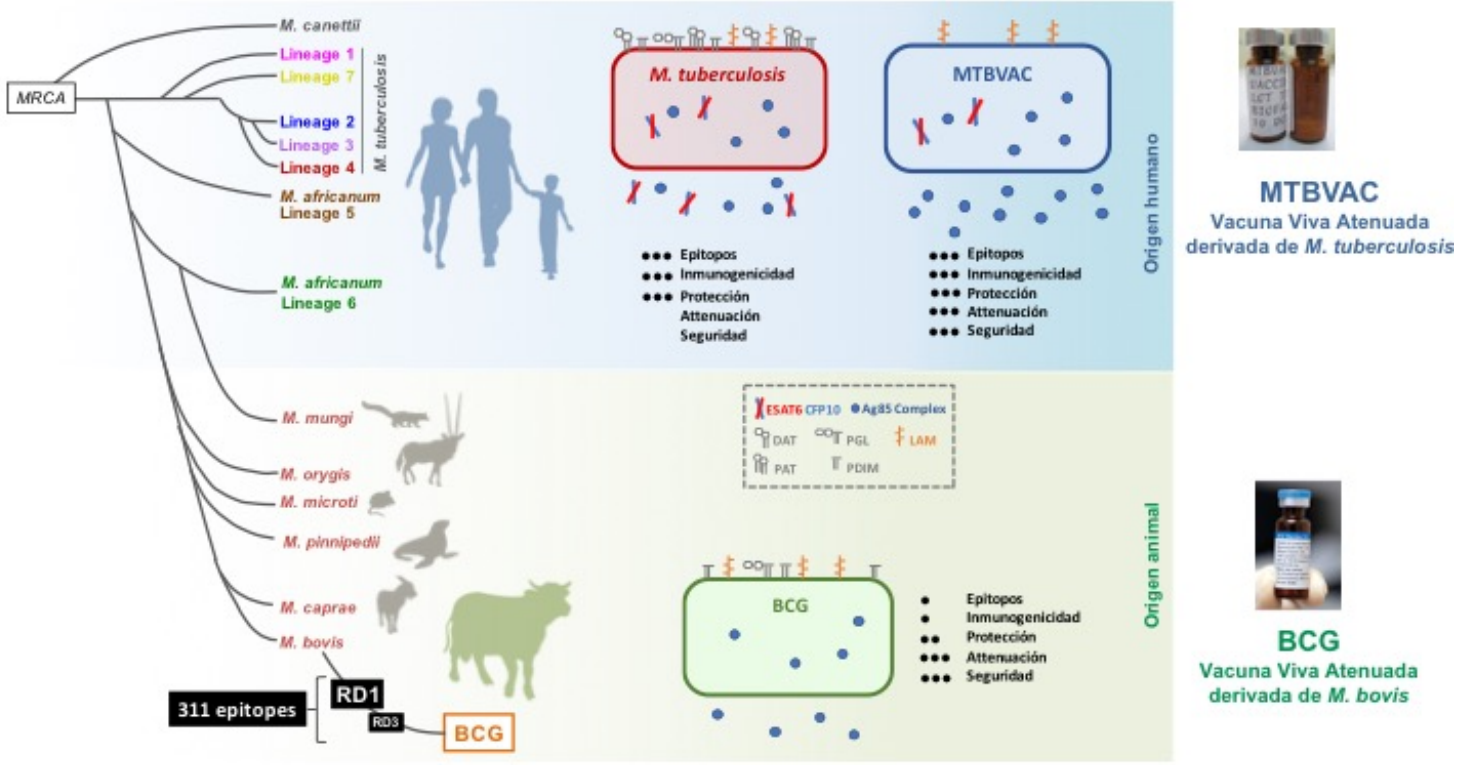

Figura 6 Diferencias entre MTBVAC y BCG. La figura muestra la representación de M. tuberculosis, BCG y MTBVAC. BCG ha perdido la región diferencial RD1 que ha llevado a su atenuación. MTBVAC contiene el repertorio antigénico de $M$. tuberculosis ausente en BCG incluido RD1, y que contiene más de 300 epitopos y al mismo tiempo presentar un perfil de atenuación comparable al BCG. 